\title{
Adaptive evolution in invasive species
}

\author{
Peter J. Prentis ${ }^{1}$, John R.U. Wilson ${ }^{2}$, Eleanor E. Dormontt ${ }^{1}$, David M. Richardson ${ }^{2}$ \\ and Andrew J. Lowe ${ }^{1,3}$
}

\author{
${ }^{1}$ Australian Centre for Evolutionary Biology and Biodiversity, School of Earth and Environmental Sciences, University of Adelaide, \\ North Terrace, South Australia 5005, Australia \\ ${ }^{2}$ Centre for Invasion Biology, Department of Botany and Zoology, Stellenbosch University, Private Bag X1, Matieland, 7600, \\ South Africa \\ ${ }^{3}$ State Herbarium and Bioknowledge South Australia, Hackney Road, Adelaide, South Australia 5005, Australia
}

\begin{abstract}
Many emerging invasive species display evidence of rapid adaptation. Contemporary genetic studies demonstrate that adaptation to novel environments can occur within 20 generations or less, indicating that evolutionary processes can influence invasiveness. However, the source of genetic or epigenetic variation underlying these changes remains uncharacterised. Here, we review the potential for rapid adaptation from standing genetic variation and from new mutations, and examine four types of evolutionary change that might promote or constrain rapid adaptation during the invasion process. Understanding the source of variation that contributes to adaptive evolution in invasive plants is important for predicting future invasion scenarios, identifying candidate genes involved in invasiveness, and, more generally, for understanding how populations can evolve rapidly in response to novel and changing environments.
\end{abstract}

\section{Introduction}

Biological invasions are defined as the introduction, establishment, and spread of species outside their native range ([1]; Figure 1]), and they are recognized as a major threat to the economy and environment worldwide [2]. By definition, introduced species are present in biogeographic regions where they did not evolve and to which they might be poorly adapted $[1,3]$, encountering a suite of novel stresses and selection pressures [4]. Consequently, introduced populations have considerable potential for rapid adaptation, and it has been proposed that this adaptation can explain why some introduced species establish, proliferate, and become invasive in new environments [1-7].

Evolutionary change was first recognized as an important process in biological invasions more than 30 years ago [8]. Nevertheless, most research in this area has been conducted in the past decade, and has concentrated on comparing genetic diversity between source and invasive populations [5]. Fewer studies have examined the role of rapid adaptive evolution in biological invasion $[7,9]$, and no study that we know of has attempted to identify the source of genetic and epigenetic variation underlying rapid adaptation in invasive species. The literature on non-invasive model or agronomic plant species, however, details a range of mechanisms that can influence rapid evolutionary change within 20 generations or fewer [10-13]. This includes a case in which heritable genomic change in

Corresponding author: Prentis, P.J. (peter.prentis@adelaide.edu.au).
Arabidopsis thaliana (thale cress) was caused by novel environmental stress [11], a mechanism not yet examined in the rapid evolution of invasive species. Given the potential that these mechanisms have for explaining invasiveness, the study of adaptive evolution in invasive species will fast become a very productive area of research.

In this review, we explore the role of rapid evolutionary change as a means of adaptation during plant invasions, and identify key research gaps. Our primary focus is to elucidate how different forms of genetic variation affect the likelihood of adaptive evolution during the introduction, establishment, and spread of invasive species (Figure 1). We examine the potential for adaptive evolution in invasive species provided by standing genetic variation compared to that from new mutations, and look at four types of evolutionary change that might promote rapid evolution in the introduced range: bottlenecks, hybridization, polyploidy, and stress-induced modification of the genome. Although adaptation from standing genetic variation and the four types of evolutionary change are not exclusive to plant invasions, the nature of many biological invasions predisposes invasive plants to these mechanisms [1,3,4]. Moreover, recent biological invasions such as that of Hypericum canariense (Canary Island St. John's wort [14]), provide an ideal opportunity to investigate rapid adaptation [15]. We advocate the use of a combined

\section{Glossary}

Additive genetic variance: variance associated with the average outcome of substituting one allele with another.

Epigenetic: a factor that changes the phenotype of an organism that is not associated with a change in its DNA sequence.

Epistasis: the phenotypic consequence of interaction among alleles at multiple loci.

Founder effect: the difference in gene pools between an original population and a new population after colonization.

Genetic bottleneck: when population numbers are reduced to a leve insufficient to sustain the diversity in the population.

Introduced species (synonyms: alien, exotic, non-indigenous, non-native): a species that is not native to a region and that was introduced to that region through human activity.

Invasive species: an introduced species that has overcome dispersal barriers to produce established self-perpetuating populations far from the initial introduction.

Polyploid: having more than two full copies of each chromosome.

Quantitative trait locus: the location on a chromosome of a gene(s) that affects a quantitative trait.

Retrotransposon: a transposon copied from RNA with the use of reverse transcriptase.

Standing genetic variation: the presence of more than one allele at a locus in a natural population.
} 


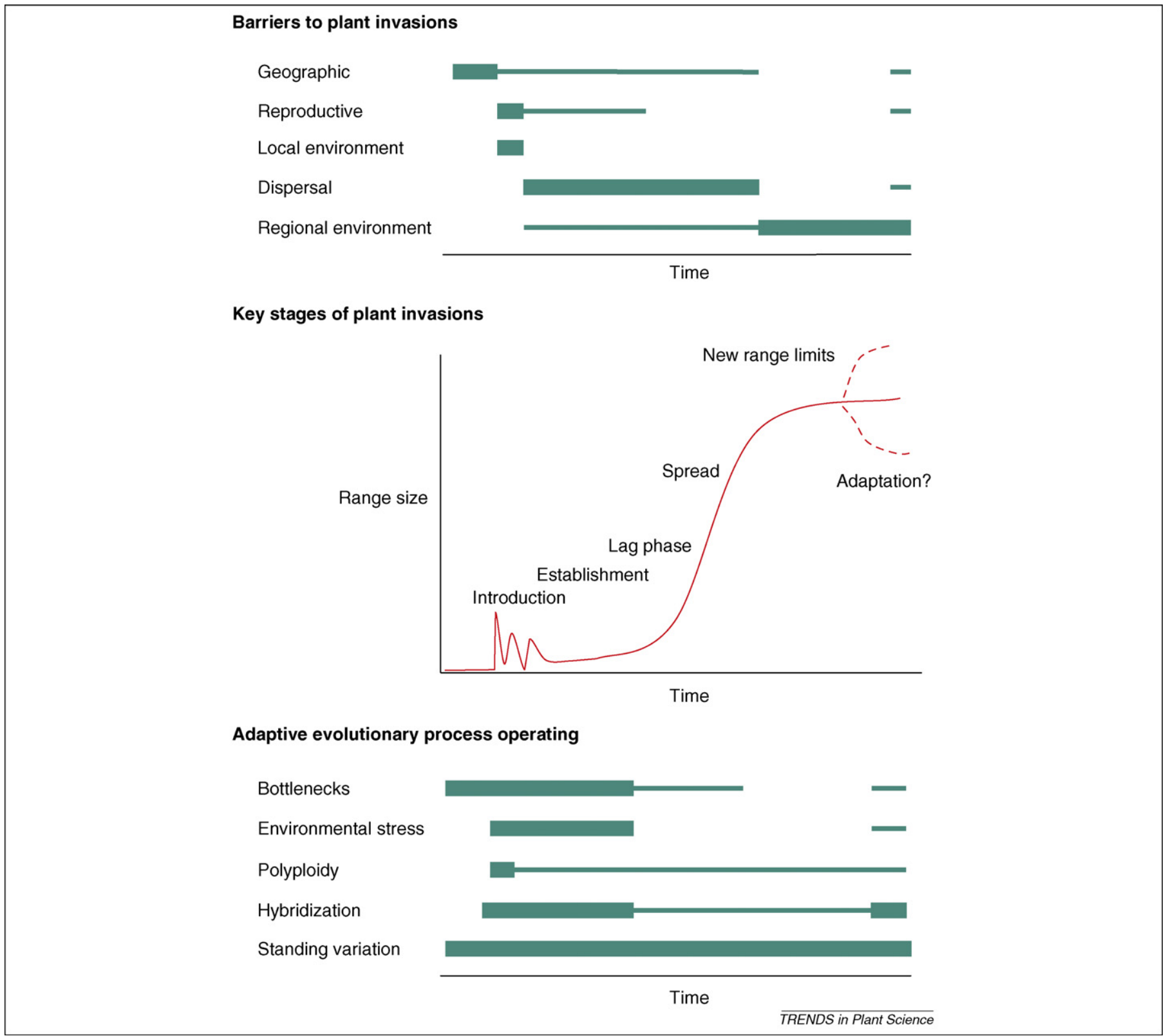

Figure 1. Adaptive evolution during the stages of invasion. Key stages in plant invasions can be depicted with regard to the change in range size over time (centre), or with reference to sequential barriers that the species must negotiate [66] (top). The barriers to invasion predispose plants to certain types of evolutionary change at different points in the invasion process (bottom). Elucidation of these dynamics is crucial for understanding adaptive evolution in plant invasions. Rapid adaptation from standing genetic variation is likely at all stages of invasion [18], but other evolutionary processes will be important at particular stages. Bottlenecks are likely to be particularly prevalent during the introduction and establishment stages because of founder effects and population isolation after dispersal involving a long-distance jump [14,24]. Evolutionary change induced by new competitors, pathogens, natural enemies, or abiotic stressors, is most likely to occur during introduction and spread. Polyploidy can benefit plants during early invasion if individuals with higher ploidy levels are stronger competitors or better colonizers than diploids [49,50]. Hybridisation can have immediate impacts on invasive potential in neo-allopolyploids because of fixed heterosis [51], but might also be important in establishment and spread if adaptive introgression and transgressive segregation aid the colonization of new environments $[9,37]$. Note, the success or sudden decline [67,68] of invasive plants can also be caused by adaptive evolution in herbivores, pollinators, fungal symbionts or competing plants.

quantitative trait locus (QTL) mapping and genome scan approach [16] to discover the underlying genetic changes associated with successful invasions and potentially to identify candidate genes that are involved in invasiveness.

\section{Standing genetic variation compared with new mutation}

Whether the genetic changes that allow species adapt to new environments arise from new mutation or standing genetic variation is currently a key question in evolutionary biology [17], and this question is equally important for understanding the rapid evolution of invasiveness. We predict that, during the invasion process, rapid adaptation, occurring over tens of generations or fewer [13], should mostly be due to alleles from standing genetic variation because favourable alleles are immediately available and are usually found at a greater frequency in populations relative to neutral or deleterious alleles [18]. Moreover, because invasive populations frequently face new environmental conditions, alleles that have neutral or even deleterious effects in the native range can become advantageous in the introduced range. Evidence supporting our prediction comes from the natural invasion of the Caribbean islands and Central America from Brazil by 
Eichhornia paniculata (Brazilian water hyacinth). In this species, recessive modifier genes that promote selfing often occur at low frequencies in outcrossing Brazilian populations but fail to spread, possibly as reliable pollination services are available and because of genetic costs associated with inbreeding depression [19,20]. Self-fertilization has evolved, however, in the Caribbean islands where the recessive modifier genes have increased in frequency [21], possibly because of unreliable pollinator services in this new environment. Adaptation from standing genetic variation should also dominate rapid evolution during range expansion, particularly when invasive species are exposed to ecogeographic variation. Geographical clines in lifehistory traits that have been found in many invasive plants, including California poppies (Eschscholzia californica) and Purple loosestrife (Lythrum salicaria), provide compelling evidence of local adaptation to environmental conditions [20,22]. Clines observed in $E$. californica and $L$. salicaria mirror similar clines observed in the native range of these plants, indicating that this adaptation possibly arose from standing genetic variation.

Recent advances in population genetic theory $[17,18,23]$ are making it possible to distinguish between adaptation from standing genetic variation and adaptation from new mutation, as they leave different molecular signatures in the genome. By applying this theory in conjunction with genome scans, it will become possible to decipher whether rapid evolution in invasive species is mainly through selection on standing genetic variation.

\section{Bottlenecks}

Genetic bottlenecks are commonly predicted to be associated with biological invasions [24], as introduced populations can be founded by a small number of individuals that are isolated from further gene flow [14]. Whether genetic bottlenecks constrain the speed of rapid adaptation is a question of great interest to invasion biologists $[5,13,14]$. Traditionally, genetic bottlenecks were thought to decrease the potential for adaptive evolution [25] because of a reduction in the quantitative variation within a population. Recent theoretical research, however, has suggested that bottlenecks can lead to increased genetic variance in founding populations because of the conversion of epistatic to additive variance [26], and hence, bottlenecks could promote rapid adaptive evolution. Conversely, Turelli and Barton [27] argue that biologically realistic models do not predict large conversions of epistatic to additive variance following a bottleneck. They argue that epistatic components of variance for most traits tend to be small [28] and that an increase in variance is appreciable only under extreme levels of inbreeding. In view of these arguments, we predict that bottlenecks restrict the speed of rapid adaptive evolution during most introductions, but have the potential to promote rapid adaptation under special conditions.

A particularly interesting example of rapid adaptive evolution after a strong genetic bottleneck is provided by H. canariense, Canary Island St. John's wort. Three isolated invasive populations showed evidence of a strong genetic bottleneck (expected heterozygosity was $45 \%$ lower than that of native populations). Despite this loss of genetic variation, the three introduced populations had higher growth rates than source populations, and a cline in flowering time has developed among locations, indicating adaptation to local environmental conditions in less than 50 years. It is not known, however, whether adaptation would have been quicker had there been no bottleneck.

\section{Hybridization}

Hybridization, both intra- and interspecific, has been suggested to stimulate invasiveness in plants [29]. Mounting evidence is becoming available to support this claim because many invading species, including Ambrosia artemisiifolia (Common ragweed) and Cytisus scoparius (Scotch broom), have multiple introductions. Other invading taxa, such as Helianthus annuus ssp. texanus (Weedy sunflower) are the by-products of inter-specific hybridization $[5,9,14,30-33]$. In some cases, however, multiple invasion events can lead to a mosaic of maladaptation, where trait values from one population might be better suited in another population, a pattern observed for Verbascum thapsus (Common mullein) [14].

Invasion biologists are exceptionally interested in finding out what types of hybridisation provide the genetic novelty for rapid adaptation. A hybrid might have increased fitness due to heterosis or later-generation recombination of parental genotypes [34]. First-generation hybrids often exhibit heterosis, and are larger and have increased yield compared to parental lines [35]. With the exception of asexual species and allopolyploids [36], however, the benefits of heterosis diminish in later-generation hybrids because of recombination and reduction in heterozygosity. Because heterosis can be transient, we predict that recombination is more important in the rapid evolution of invasive species.

Recombination can generate novel gene combinations upon which natural selection acts to produce a phenotype that is better suited to colonise novel environments [37]. Senecio squalidus (Oxford ragwort) provides a classic example of this. S. squalidus, a recombinant hybrid between Senecio aethensis and Senecio chrysanthemumifolius, was originally brought from Mount Etna in Sicily to Oxford Botanical Garden, England. Both parental species are absent from the wild in the UK, but the hybrid species became invasive and is now widespread [38].

Enhanced invasiveness as the result of recombination can occur by two main mechanisms. First, hybrids can exhibit traits that are novel or extreme relative to those of either parental line, a common feature of plant hybrids in particular $[39,40]$. Such extreme phenotypes are often the outcome of transgressive segregation, where alleles from both parents combine to produce extreme traits that exceed parental phenotypic values in either a negative or positive direction [34]. In a recent study of gene expression using a non-invasive hybrid species, Helianthus deserticola (Desert sunflower), and its parental species, $2 \%$ of genes exhibited transgressive expression profiles [41]. H. deserticola has colonized a novel habitat type not inhabited by either parent, indicating that transgressive segregation associated with interspecific hybridization can aid the colonization of novel environments. In some cases, transgressive gene expression in interspecific hybrids results in 
changes to patterns of parental gene expression that are so extensive that it has been termed 'transcriptome shock' [42].

Second, hybridization can cause adaptive trait introgression, in which alleles that could potentially increase fitness or fecundity are transferred from one species to another [9]. Adaptive trait introgression has conferred herbivore-resistant traits from Beach sunflower, Helianthus debilis, into common sunflower, Helianthus annuus ssp. annuus. This introgression has allowed a stabilized hybrid lineage of this species, $H$. annuus ssp. texanus, to colonize new environments in central and southern Texas [9]. In two uniform environment experiments located in southern Texas, $H$. annuus ssp. texanus exhibited 55\% higher fitness than $H$. annuus ssp. annuus and was more resistant to herbivores.

\section{Polyploidy}

Polyploidy or genome doubling has been a pervasive force in plant evolution [43]. Interestingly, polyploids occur with greater frequency among invasive plants than among angiosperms in general [44,45]. In many cases where species consist of two or more ploidy levels in the native range, invasive populations often only contain individuals of the higher ploidy level, for example Fallopia japonica (Japanese knotweed) in Europe [46]. Alternatively, invasive populations can be neo-allopolyploids, such as Tragopogon mirus (remarkable Goat's beard) [47], formed from recent hybridization between two species and subsequent genome doubling. Why polyploids are overrepresented on lists of invasive species is currently unknown, and different explanations probably apply for neo-allopolyploids and other polyploids. One reason for the greater incidence of invasiveness among taxa that have higher ploidy levels is that there might be fitness or competitive difference(s) between populations with different ploidy levels in some species complexes. For example, Flegrova and Krahulec [48] found, using reciprocal transplant experiments, that tetraploid Anthoxanthum odoratum (Sweet vernal grass) had higher fitness than diploid Anthoxanthum alpinum (Alpine sweet vernal grass) in both habitats. Another reason for the high incidence of invasiveness among polyploids could be that they are better colonizers than diploids, a pattern that has received some support [49], particularly in the Artic flora [50].

Neo-allopolyploids are probably successful invaders because of fixed heterosis that results from the fixing of divergent parental genomes in allopolyploids. The enforced pairing of homologous chromosomes in allopolyploids prevents intergenomic recombination, and hence the benefits of heterosis are not diminished in later generations of allopolyploid hybrids [51]. Fixed heterosis is probably the major reason for the success of neo-allopolyploids that reproduce asexually. Neo-allopolyploids that reproduce sexually, however, often pass through strong fertility bottlenecks associated with difficulties in meiosis [52,53], which might slow adaptation.

Allopolyploids can also undergo extensive alterations to their genome and transcriptome, including some that arise with the onset of genome doubling [43]. Novel phenotypes are known to emerge from these changes, including some with high visibility to selection [43], and these changes might promote invasiveness in some neo-allopolyploids. Recent studies demonstrate, however, that hybridization has a greater effect than chromosome doubling on genomic changes in allopolyploids [42]. Thus, hybridization could be more important for the success of allopolyploid invaders than is genome doubling.

\section{Stress-induced modification of the genome}

Invasive species frequently encounter novel ecological contexts [4], both biotic (e.g. pathogens) and abiotic (i.e. UV exposure). Exposure to novel biotic or abiotic conditions can induce stress in some plants, and has been shown to affect genome stability in some cases $[54,55]$. This process is, on initial inspection, akin to the ideas of Lamarck, but a recent review distinguishes this area as stress-induced modification of the genome [12]. Stress-induced genome modification can be epigenetic [11]; alternatively, it can occur when retrotransposons activate and reinsert into the genome [56] and in some instances are stably inherited $[54,57]$.

Stress-induced genomic effects have been implicated in rapid genomic or phenotypic change in several well-studied plant species, including Arabidopsis and flax (Linum usitatissimum) [10,11,55]. For example, elevated UV-C exposure and pathogen attack increased rates of homologous recombination in Arabidopsis for at least four generations in the progeny of treated plants [11]. Abiotic stress has also been shown to activate retrotransposons in Triticum (wheat), resulting in the silencing of genes adjacent to the insertion sites of the retrotransposons and altering the expression patterns of these genes [56]. A particularly interesting example of phenotypic change promoted by novel environmental stress occurs in L. usitatissimum, in which growth under different fertilizer treatments resulted in large-scale genomic changes in one generation (see Figure 2 for a detailed description of this process). These examples show that a decrease in genome stability, in response to environmental stimuli, can cause modifications to the genome and transcriptome, resulting in new phenotypic variation that is then exposed to selection $[12,55]$. Although stress-induced modification of the genome has only recently emerged as a mechanism that can create genetic novelty, we predict that it will explain some instances of rapid adaptation in invasive species.

\section{A genomic approach to studying adaptive evolution in invasive species}

Understanding the genetic basis of traits that are involved in rapid adaptation to novel environmental conditions is a major goal in invasion biology. In particular, identifying the genes or genetic changes that underlie these traits will permit us to answer fundamental questions: how often does rapid adaptation result from standing genetic variation rather than new mutation in successful plant invasions? And are similar genes the targets of selection when the same species undergoes multiple independent invasions? To answer these questions, and to identify genes underlying invasive traits, we recommend an approach put forward recently by Stinchcombe and Hoekstra [16]. This powerful approach uses a genome scan in combination with 


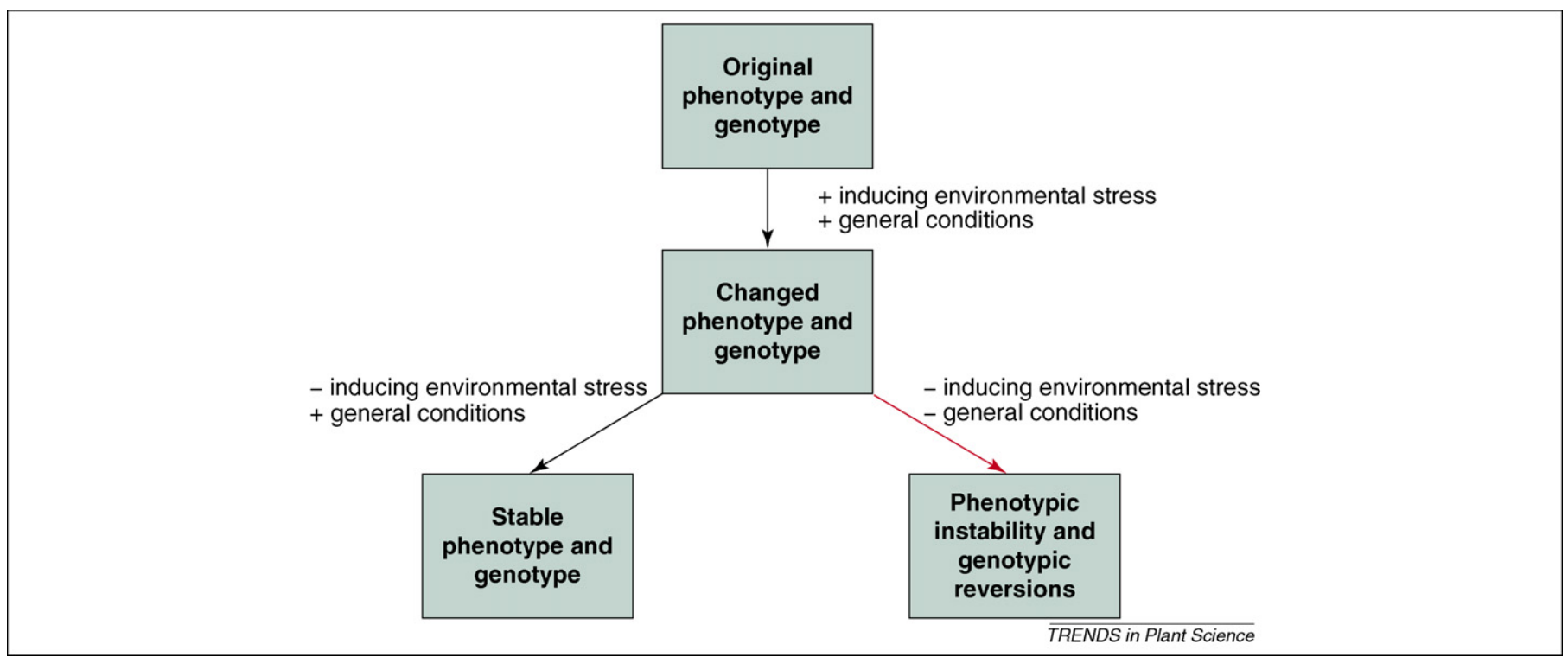

Figure 2. Environmentally induced heritable change observed in flax. In Linum usitatissimum (flax), growth on different fertilizer treatments (with or without environmental

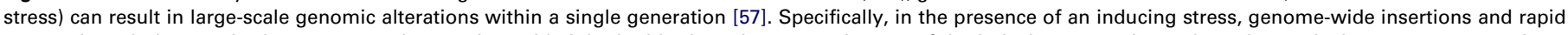
genotypic and phenotypic changes occur that can be stably inherited in the subsequent absence of the inducing stress, but only under particular temperature regimes (general conditions) [55]. Without the general conditions, the phenotypes and genotypes of subsequent generations can be unstable. Interestingly, this instability can be stopped at any point by resuming the presence of general conditions during early growth. Recent work has identified a specific sequence (LIS-1) that is consistently inserted into the genome at the same site and at the same growth stage when flax is subjected to the initial inducing stress; this is thought to involve a complex set of insertions and rearrangements and is probably typical of similar changes occurring throughout the flax genome [57].

QTL mapping to identify chromosomal regions and genes within these regions that are involved in adaptive evolution in invasive plants.

Genome scans involve genotyping numerous loci throughout the genome of many individuals in several populations [58] to detect 'outlier' loci that show unusually high levels of differentiation. This makes it possible to distinguish between evolutionary forces that affect the whole genome (e.g. bottlenecks) and those that affect particular loci (i.e. selection) [59]. For non-model species whose genomes have not been sequenced, which include most invasive plants, the most effective genome scan approach to identify 'outliers' utilizes microsatellites in the untranslated regions of expressed sequence tags [60]. This approach makes use of close linkage between a polymorphic marker and a transcribed gene to detect candidate loci that might be involved in adaptive evolution. By scanning the genomes of populations from the native and introduced range of invasive species, we will be able to determine both the severity of bottlenecks at population founding and potential candidate loci that have been the target of selection. It is important to note, however, that genome scans have a major limitation when used in isolation: it is often unclear which 'outlier' loci underlie phenotypic traits of interest.

QTL mapping uses statistical analyses of both molecular markers that are distributed throughout the genome and traits measured in the progeny of controlled crosses to identify stretches of DNA that are closely linked to genes that underlie the trait in question [16]. Thus, QTL studies have been useful for estimating the complexity of the genetic architecture that underlies phenotypic traits in many plant species, from Quercus robur and Quercus petraea (a closely related oak species) to Arabidopsis
[61,62]. Using a QTL approach on a mapping population generated from a cross between the native and introduced range of an invasive species, it should be possible to identify the position and number of genes that control traits that are important to successful invasions [63]. QTL approaches provide insight into the nature of adaptation to different environmental conditions, but they seldom lead to the genes underlying particular traits because a given QTL typically contains many genes. Nevertheless, there are exceptions in which specific genes that are linked to a particular trait have been identified [64].

Combining a genome scan with QTL mapping has advantages over either approach taken in isolation. For instance, outlier loci identified from genome scans can be integrated onto linkage maps to determine if they map to chromosomal regions underlying traits of interest. This approach has been used with success in Zea mays ssp. mays (maize) and its wild progenitor $Z$. mays ssp. parviglumis (Teosinte) to identify genes that were possible targets of selection during domestication [65]. In this study, two outlier loci were found to map close to a chromosomal region controlling two traits that differ significantly between maize and teosinte. We predict that utilizing this combined approach can move the field of invasion biology forward by allowing researchers to determine the genetic basis of invasiveness, and in some instances, to identify candidate genes or mutations that are involved in the rapid evolution of invasiveness.

\section{Acknowledgements}

We thank three anonymous reviewers for insightful comments that greatly improved this manuscript and Ana Pavasovic for help with Figure 2. This work was supported in part by the ARC Discovery Grant (DP0664967) awarded to A.J.L. by the DST-NRF Centre of Excellence for Invasion Biology and the Working for Water Programme. 


\section{References}

1 Richardson, D.M. and Pyšek, P. (2006) Plant invasions: merging the concepts of species invasiveness and community invasibility. Prog. Phys. Geog 30, 409-431

2 Pimentel, D. et al. (2005) Update on the environmental and economic costs associated with alien-invasive species in the United States. Ecol. Econ. 52, 273-288

3 Facon, B. et al. (2006) A general eco-evolutionary framework for understanding bioinvasions. Trends Ecol. Evol. 21, 130-135

4 Novak, S.J. (2007) The role of evolution in the invasion process. Proc. Natl. Acad. Sci. U. S. A. 104, 3671-3672

5 Bossdorf, O. et al. (2005) Phenotypic and genetic differentiation between native and introduced plant populations. Oecologia 144, 1-11

6 Maron, J.L. et al. (2004) Rapid evolution of an invasive plant. Ecol. Monogr. 74, 261-280

7 Lavergne, S. and Molofsky, J. (2007) Increased genetic variation and evolutionary potential drive the success of an invasive grass. Proc. Natl. Acad. Sci. U. S. A. 104, 3883-3888

8 Baker, H.G. (1974) The evolution of weeds. Annu. Rev. Ecol. Syst. 5, 124

9 Whitney, K.D. et al. (2006) Adaptive introgression of herbivore resistance traits in the weedy sunflower Helianthus annuus. Am. Nat. 167, 794-807

10 Alleman, M. et al. (2006) An RNA-dependent RNA polymerase is required for paramutation in maize. Nature 442, 295-298

11 Molinier, J. et al. (2006) Transgeneration memory of stress in plants. Nature 442, 1046-1049

12 Rando, O.J. and Verstrepen, K.J. (2007) Timescales of genetic and epigenetic inheritance. Cell 128, 655-668

13 Hendry, A.P. et al. (2007) The speed of ecological speciation. Funct. Ecol. 21, 455-464

14 Dlugosch, K.M. and Parker, I.M. (2008) Founding events in species invasions: genetic variation, adaptive evolution, and the role of multiple introductions. Mol. Ecol. 17, 431-449

15 Sax, D.F. et al. (2007) Ecological and evolutionary insights from species invasions. Trends Ecol. Evol. 22, 465-471

16 Stinchcombe, J.R. and Hoekstra, H.E. (2008) Combining population genomics and quantitative genetics: finding the genes underlying ecologically important traits. Heredity 100, 158-170

17 Hermisson, J. and Pennings, P.S. (2005) Soft sweeps: molecular population genetics of adaptation from standing genetic variation. Genetics 169, 2335-2352

18 Barrett, R.D.H. and Schluter, D. (2008) Adaptation from standing genetic variation. Trends Ecol. Evol. 23, 38-44

19 Fenster, C.B. and Barrett, S.C.H. (1994) Inheritance of mating-system modifier in Eichhornia paniculata. Heredity 72, 433-445

20 Barrett, S.C.H. et al. (2008) Plant reproductive systems and evolution during biological invasion. Mol. Ecol. 17, 373-383

21 Kollmann, J. and Bañuelos, M.J. (2004) Latitudinal trends in growth and phenology of the invasive alien plant Impatiens glandulifera (Balsaminaceae). Divers. Distrib. 10, 377-385

22 Leger, E.A. and Rice, K.J. (2003) Invasive California poppies (Eschscholzia californica Cham.) grow larger than native individuals under reduced competition. Ecol. Lett. 6, 257-264

23 Przeworski, M. et al. (2005) The signature of positive selection on standing genetic variation. Evolution Int. J. Org. Evolution 59, 2312-2323

24 Barrett, S.C.H. and Richardson, B.J. (1986) Genetic attributes of invading species. In Ecology of Biological Invasions: An Australian Perspective (Groves, R.H. and Burdon, J.J., eds), pp. 21-33, Australian Academy of Science

25 Van Buskirk, J. and Willi, Y. (2006) The change in quantitative genetic variation with inbreeding. Evolution Int. J. Org. Evolution 60, 2428 2434

26 Naciri-Graven, Y. and Goudet, J. (2003) The additive genetic variance after bottlenecks is affected by the number of loci involved in epistatic interactions. Evolution Int. J. Org. Evolution 57, 706-716

27 Turelli, M. and Barton, N.H. (2006) Will multilocus epistasis and population bottlenecks increase additive genetic variance? Evolution Int. J. Org. Evolution 60, 1763-1776

28 Whitlock, M.C. et al. (1995) Multiple fitness peaks and epistasis. Annu. Rev. Ecol. Syst. 26, 601-629
29 Ellstrand, N.C and Schierenbeck, K. (2000) Hybridization as a stimulus for the evolution of invasiveness in plants? Proc. Natl. Acad. Sci. U. S. A. 97, 7043-7050

30 Petit, R.J. et al. (2004) Ecology and genetics of tree invasions: from recent introductions to Quaternary migrations. Forest Ecol. Manag. 197, 117-137

31 Genton, B.J. et al. (2005) High genetic diversity in French invasive populations of common ragweed, Ambrosia artemisiifolia, as a result of multiple sources of introduction. Mol. Ecol. 14, 4275-4285

32 Muirhead, J.R. et al. (2008) Identifying the source of species invasions: sampling intensity versus genetic diversity. Mol. Ecol. 17, 1020-1035

33 Kang, M. et al. (2007) Testing the role of genetic factors across multiple independent invasions of the shrub Scotch broom (Cytisus scoparius). Mol. Ecol. 16, 4662-4673

34 Baack, E.J. and Rieseberg, L.H. (2007) A genomic view of introgression and hybrid speciation. Curr. Opin. Genet. Dev. 17, 513-518

35 Hochholdinger, F. and Hoecker, N. (2007) Towards the molecular basis of heterosis. Trends Plant Sci. 12, 427-432

36 Abbott, R.J. and Lowe, A.J. (2004) Origins, establishment and evolution of new polyploid species: Senecio cambrensis and $S$. eboracensis in the British Isles. Biol. J. Linn. Soc. 82, 467-474

37 Rieseberg, L.H. et al. (2003) Major ecological transitions in wild sunflowers facilitated by hybridization. Science 301, 1211-1216

38 James, J.K. and Abbott, R.J. (2005) Recent, allopatric, homoploid hybrid speciation: the origin of Senecio squalidus (Asteraceae) in the British Isles from a hybrid zone on Mount Etna, Sicily. Evolution Int. J. Org. Evolution 59, 2533-2547

39 Lowe, A.J. and Abbott, R.J. (2004) Reproductive isolation of a new hybrid species, Senecio eboracensis Abbott \& Lowe (Asteraceae). Heredity 92, 386-395

40 Arnold, M.L. and Hodges, S.A. (1995) Are natural hybrids fit or unfit relative to their parents? Trends Ecol. Evol. 10, 67-70

41 Lai, Z. et al. (2006) Microarray analysis reveals differential gene expression in hybrid sunflower species. Mol. Ecol. 15, 1213-1227

42 Hegarty, M.J. et al. (2006) Transcriptome shock after interspecific hybridization in Senecio is ameliorated by genome duplication. Curr. Biol. 16, 1652-1659

43 Adams, K.L. and Wendel, J.F. (2005) Novel patterns of gene expression in polyploid plants. Trends Genet. 21, 539-543

44 Brown, A.H.D. and Marshall, D.S. (1981) Evolutionary changes accompanying colonization in plants. In Evolution Today: Proceedings of $2^{\text {nd }}$ International Congress of Systematics and Evolutionary Biology (Scudder, G.G.E. and Reveal, J.L., eds), pp. 351-363, Carnegie-Mellon University

45 Pandit, M.K. et al. (2006) Polyploidy in invasive plant species from Singapore. Bot. J. Linn. Soc. 151, 395-403

46 Hollingsworth, M.L. and Bailey, J.P. (2000) Evidence for massive clonal growth in the invasive weed Fallopia japonica (Japanese knotweed). Bot. J. Linn. Soc. 133, 463-472

47 Novak, S.J. et al. (1991) Ownbey's Tragopogons: 40 years later. Am. J. Bot. 78, 1586-1600

48 Flegrova, M. and Krahulec, F. (1999) Anthoxanthum odoratum and A. alpinum: life history parameters at two different altitudes. Folia Geobot. 34, 19-31

49 Soltis, P.S. and Soltis, D.E. (2000) The role of genetic and genomic attributes in the success of polyploids. Proc. Natl. Acad. Sci. U. S. A. 97, 7051-7057

50 Brochmann, C. et al. (2004) Polyploidy in Artic plants. Biol. J. Linn. Soc. $82,521-536$

51 Comai, L. (2005) The advantages and disadvantages of being polyploid Nat. Rev. Genet. 6, 836-846

52 Ramsey, J. and Schemske, D.W. (2002) Neopolyploidy in flowering plants. Annu. Rev. Ecol. Syst. 33, 589-639

53 Madlung, A. et al. (2005) Genomic changes in synthetic Arabidopsis polyploids. Plant J. 41, 221-230

54 Bond, D.M. and Finnegan, E.J. (2007) Passing the message on: inheritance of epigenetic traits. Trends Plant Sci. 12, 211-216

55 Henikoff, S. (2005) Rapid changes in plant genomes. Plant Cell 17, 2852-2855

56 Kashkush, K. et al. (2003) Transcriptional activation of retrotransposons alters the expression of adjacent genes in wheat. Nat. Genet. 33, 102-106 
57 Cullis, C.A. (2005) Mechanisms and control of rapid genomic changes in flax. Ann. Bot. (Lond) 95, 201-206

58 Luikart, G. et al. (2003) The power and promise of population genomics: from genotyping to genome typing. Nat. Rev. Genet. 4, 981-994

59 Beaumont, M.A. and Balding, D.J. (2004) Identifying adaptive genetic divergence among populations from genome scans. Mol. Ecol. 13, 969980

60 Bouck, A. and Vision, T. (2007) The molecular ecologist's guide to expressed sequence tags. Mol. Ecol. 16, 907-924

61 Weinig, C. et al. (2003) Heterogeneous selection at specific loci in natural environments of Arabidopsis thaliana. Genetics 165, 321-329

62 Scotti-Saintagne, C. et al. (2004) Genome scanning for interspecific differentiation between two closely related oak species. Genetics 168, $1615-1626$

63 Weinig, C. et al. (2007) Resolving the genetic basis of. invasiveness and predicting invasions. Genetica 129, 205-216
64 Balasubramanian, S. et al. (2006) The PHYTOCHROME C photoreceptor gene mediates natural variation in flowering and growth responses of Arabidopsis thaliana. Nat. Genet. 38, 711-715

65 Vigouroux, Y. et al. (2002) Identifying genes of agronomic importance in maize by screening microsatellites for evidence of selection during domestication. Proc. Natl. Acad. Sci. U. S. A. 99, 9650-9655

66 Richardson, D.M. et al. (2000) Naturalization and invasion of alien plants: concepts and definitions. Divers. Distrib. 6, 93-107

67 Simberloff, D. and Gibbons, L. (2004) Now you see them, now you don't - population crashes of established introduced species. Biol. Invasions $6,161-172$

68 Hawkes, C.V. (2007) Are invaders moving targets? The generality and persistence of advantages in size, reproduction, and enemy release in invasive plant species with time since introduction. Am. Nat. 170, 832843

\section{Plant Science Conferences in 2008}

18th International Plant Lipid Symposium

20-25 July 2008

Bordeaux, France

http://www.ispl2008-bordeaux.cnrs.fr

Banff Conference on Plant Metabolism

2008 July 30 - August 3, 2008

Banff, Alberta, Canada

http://www.ucalgary.ca/plantmetabolism

XVI Congress of the Federation of European Societies of Plant Biology (FESPB 2008)

17-22 August 2008

Tampere, Finland

http://www.fespb2008.org/

Gordon Research Conference

CO2 Assimilation in Plants: Genome to Biome

17-22 August 2008

University of New England

Biddeford, Maine

www.grc.uri.edu/programs/2008/co2.htm 